Informe especial

\title{
La hospitalización domiciliaria: antecedentes, situación actual y perspectivas
}

\author{
Rosângela Minardi Mitre Cotta, ${ }^{1,2,3}$ María Morales Suárez-Varela, ${ }^{1,3}$ \\ Agustín Llopis González, ${ }^{1,3}$ José Sette Cotta Filho, ${ }^{4}$ \\ Enrique Ramón Real ${ }^{5}$ y José Antonio Días Ricós ${ }^{5}$
}

RESUMEN Objetivo. Determinar las distintas variantes que ha tenido el concepto de la hospitalización domiciliaria a lo largo del tiempo, para así contribuir al debate sobre las circunstancias que inciden en la gestión sanitaria frente a futuros retos.

Métodos. Se revisa la literatura sobre la atención domiciliaria como modalidad asistencial de salud mediante una exploración de las publicaciones indizadas en MEDLINE, LILACS y el Índice Médico Español durante el período de 1995-2000.

Resultados. La hospitalización domiciliaria ha tenido un desarrollo desigual a lo largo del tiempo en diferentes países y son varios los modelos de este tipo de atención, cada uno con sus respectivas ventajas y desventajas.

Conclusiones. Se definen algunos criterios y propuestas que podrían enmarcar una gestión domiciliaria innovadora, efectiva y de calidad. Se concluye que la atención domiciliaria podría contribuir a diseñar y establecer un modelo consensuado y armonioso de organización y financiación entre los niveles de atención primaria y hospitalaria.

Palabras clave Hospitalización domiciliaria, atención domiciliaria, alianzas estratégicas, gestión sanitaria, innovación en salud.

En la actualidad se presentan, cada vez con más frecuencia, propuestas alternativas a la gerencia y la organización tradicionales de los servicios de salud debido a la necesidad de que estos servicios funcionen con mayor

1 Departamento de Medicina Preventiva y Salud Pública, Universidad de Valencia, España. Dirección postal: Avenida María Ros, 26-7. ${ }^{\text {a, }} 46100-$ Burjasot, Valencia, España.

2 Departamento de Nutrição e Saúde, Universidade Federal de Viçosa, Viçosa, Minas Gerais, Brasil.

3 Unidad de Investigación Clínico-Epidemiológica, Hospital Universitario Doctor Peset, Valencia, España.

4 Departamento de Medicina, Universidad de Valencia, España.

5 Unidad de Hospitalización a Domicilio, Hospital Universitario Doctor Peset, Valencia, España. eficiencia y eficacia $(1,2)$. Por otra parte, el incremento de la demanda de servicios y del gasto para la salud, los cambios demográficos, sociales y de los patrones de morbilidad, y las constantes innovaciones en el campo de la tecnología, están estimulando la aparición y difusión de nuevas formas de asistencia y atención hospitalarias $(3,4)$.

Esta actividad innovadora da preferencia a la asistencia ambulatoria y favorece la transferencia del paciente del hospital a su entorno familiar. Sus objetivos principales son mejorar la atención del paciente y lograr una utilización más eficiente de los recursos $(5,6)$.

Entre las numerosas modalidades alternativas figuran el hospital de día, el hospital de semana, la cirugía ambulatoria, la unidad médica de corta estancia y la hospitalización domiciliaria (HD). La HD rompe con el esquema clásico de atención hospitalaria tradicional, que abarca ingreso, urgencia y consulta externa $(7,8)$.

Pese a que en la literatura hay varias definiciones de la HD como modalidad de atención, así como controversias acerca del marco en que debe funcionar, parece haber consenso en que su función primordial sería brindar al paciente en su domicilio cuidados médicos y de enfermería en igual cantidad y calidad que en el hospital (4, 9). Dicho de otra forma, la HD estaría indicada para aquellos pacientes que 
necesitan asistencia especializada sin ser ingresados en un hospital, aunque no se encuentren en condiciones de pasar al régimen ambulatorio (10).

Hay que reconocer, sin embargo, que aun cuando la HD no es precisamente una modalidad reciente - lleva en funcionamiento más de medio siglo- y el contexto económico y social impone el uso óptimo de recursos que escasean, esta sigue siendo una práctica poco difundida y con un desarrollo desigual en la mayor parte del mundo $(11,12)$. Una revisión pormenorizada de la literatura permite identificar dos situaciones interesantes. Por un lado, la existencia de una gran variedad de modelos de actuación, el desconocimiento de los pacientes y la renuencia de los médicos a prescribir esta modalidad asistencial, así como el planteamiento de ciertos problemas en torno al desarrollo de la atención primaria a domicilio en ciertos países, son factores que limitan la evolución de la HD. Por otro lado, se destaca la escasez de estudios que concretamente examinan el lugar, la aplicabilidad y las características - pacientes, organización, profesionales participantes y financiación-de esta modalidad asistencial a lo largo del tiempo y en los sistemas sanitarios que la utilizan.

Tomados en cuenta estos factores, cabe resaltar que el presente es todavía un momento oportuno para definir las bases sobre las cuáles deben organizarse los servicios de atención domiciliaria. En tal sentido, habría que avanzar y profundizar en el diseño de un modelo consensuado y equilibrado de organización y financiación, que permita el crecimiento y la consolidación de servicios de HD de calidad, y un uso más adecuado y racional de los recursos $(9,10)$.

Este estudio forma parte de un proyecto de investigación de las innovaciones en la gestión y organización de sistemas sanitarios. Su objetivo fue revisar las distintas variantes que ha tenido el concepto de hospitalización domiciliaria a lo largo del tiempo, teniendo en consideración sus diferentes modelos, conceptos y grados de desarrollo en los niveles nacional e in- ternacional. De este modo se espera contribuir al análisis y el debate de las circunstancias que inciden en la gestión de salud -en especial la hospitalaria- a la luz de los retos del futuro.

\section{Materiales y métodos}

Se procedió a realizar una revisión minuciosa de la literatura, centrada en el período de 1995-2000. La información sobre el material bibliográfico se tomó de tres bases de datos: MEDLINE (base de datos de literatura internacional, producida por la National Library of Medicine de Estados Unidos), LILACS (Literatura Latinoamericana y del Caribe en Ciencias de la Salud) ${ }^{6}$ y el IME (Índice Médico Español), base mantenida por el Centro de Documentación e Informática del Consejo Superior de Investigaciones Científicas.

De manera complementaria al estudio, se consultaron también bases de datos de revistas de salud pública de difusión nacional e internacional, entre ellas Scientific Electronic Library Online $(\mathrm{SciELO})^{7}$ y Seriados en Ciencias de la Salud (SeCS) ${ }^{8}$.

Para la consulta en las bases de datos MEDLINE y LILACS se utilizaron los términos "home hospitalization", "hospital home", "home care" y "home health care" y sus equivalentes en castellano y francés. Para consultar la base de datos de LILACS se utilizaron, ade-

\footnotetext{
6 Es una base de datos cooperativa del Sistema BIREME, que es un centro de la Organización Panamericana de la Salud (OPS), Oficina Regional de la Organización Mundial de la Salud (OMS) para las Américas. Fue fundada en 1967 mediante un convenio entre la OPS y el Gobierno del Brasil. BIREME tiene la misión de contribuir al mejoramiento de la enseñanza, la investigación y la atención de la salud en los países de América Latina y de Caribe mediante el establecimiento y la coordinación del Sistema Latinoamericano y del Caribe de Información Científica de la comunidad de profesionales de la salud (www.bireme.br).

7 SciELO: es una biblioteca en línea de revistas científicas de salud pública que ofrece acceso universal e integrado a las publicaciones relacionadas con los países de América Latina (www.scielo.org).

$8 \quad$ SeCS es una base de datos de revistas que reúne las colecciones de BIREME y de las bibliotecas de la Red Latinoamericana y del Caribe de Información en Ciencias de la Salud.
}

más, todas estas palabras clave en portugués, más "hospitalización domiciliaria", "hospitalización a domicilio" y "atención domiciliaria" en castellano y portugués.

Para la búsqueda correspondiente a la base de datos del IME (en castellano) y de las revistas en línea (en castellano, inglés y portugués), se utilizaron los términos clave "hospitalización domiciliaria", "hospitalización a domicilio" y "atención domiciliaria".

Entre los 388 artículos seleccionados, se escogieron inicialmente 156 que fueron considerados los de mayor interés para el estudio propuesto. En una tercera fase, solo 98 artículos fueron analizados detenidamente conforme a los criterios y objetivos planteados en este estudio.

\section{Reseña histórica de la hospitalización domiciliaria}

La modalidad de la HD fue inaugurada en 1947 en Nueva York, Estados Unidos, como una extensión del hospital hacia el domicilio del paciente. Según se constata en la literatura, las razones para crear esta primera unidad de HD en el mundo eran descongestionar las salas hospitalarias y brindar a los pacientes un ambiente más humano y favorable a su recuperación $(9,11-13)$. Desde entonces ha habido múltiples experiencias de este tipo tanto en Norteamérica como en Europa, con estructuras y procedimientos adaptados a cada sistema nacional de salud (12).

A partir de los años sesenta en Canadá comenzaron a funcionar servicios de HD orientados a pacientes quirúrgicos dados de alta tempranamente. En hospitales de Montreal se realizó, en 1987, una experiencia piloto (bautizada Hôpital extra-mural) que consistía en la administración y control de antibióticos parenterales en el domicilio de pacientes con problemas agudos $(9,11)$.

En Europa, el Hospital de Tenon en París, Francia, fue el primero en crear una unidad de hospitalización domiciliaria en 1951. Más tarde, en 1957, se 
estableció en la misma ciudad el Santé Service, organización no gubernamental sin fines de lucro que aún hoy sigue prestando asistencia sociosanitaria a domicilio a pacientes con padecimientos crónicos y terminales (11). (Curiosamente, la HD en Francia recién fue reconocida plena y oficialmente como una alternativa a la hospitalización tradicional desde 1992, en virtud de los decretos de octubre $[9,11]$.)

Sin embargo, la HD tardó casi una década en empezar a desarrollarse en otros países de Europa. En el Reino Unido esta modalidad asistencial fue introducida en 1965 con el nombre de Hospital Care at Home (Atención Hospitalaria en el Hogar). En Alemania y en Suecia fue desarrollada durante los años setenta, y en Italia - con el nombre de Ospedalizzacione a Domicilio-, recién a comienzos de los años ochenta $(7,9)$.

El desarrollo de la HD en Europa ha sido siempre muy irregular, tanto cualitativa como cuantitativamente. Con el objeto de contribuir en este sentido, la Oficina Europea de la Organización Mundial de la Salud coordina desde 1996 el programa From Hospital to Home Health Care (Del hospital a la atención de salud en el hogar), dirigido a promover, estandarizar y registrar más adecuadamente esta modalidad asistencial $(11,13)$.

\section{Variaciones en la hospitalización domiciliaria según los países}

Ciertos criterios generales que enmarcan la implantación y el funcionamiento de las unidades de HD en los diferentes países se explican básicamente a partir de dos puntos de vista. En Estados Unidos y Francia, la justificación inicial para establecer la HD fue aumentar la disponibilidad de camas hospitalarias y humanizar más la atención al paciente. Asimismo, otro objetivo de introducir esta modalidad asistencial ha sido simplemente reducir los costos hospitalarios $(3,4)$.

La literatura sobre el tema también indica que los países difieren en el uso de la HD según el diagnóstico de los

CUADRO 1. Tres principales diagnósticos asociados con la hospitalización domiciliaria en diferentes países

\begin{tabular}{llll}
\hline \multicolumn{1}{c}{ País } & Primer diagnóstico & Segundo diagnóstico & Tercer diagnóstico \\
\hline Canadá & $\begin{array}{c}\text { Enfermedades del } \\
\text { aparato circulatorio }\end{array}$ & Enfermedades de la piel & $\begin{array}{c}\text { Enfermedades del } \\
\text { aparato respiratorio }\end{array}$ \\
$\begin{array}{c}\text { Estados Unidos } \\
\text { de América }\end{array}$ & $\begin{array}{c}\text { Enfermedades del } \\
\text { aparato circulatorio }\end{array}$ & $\begin{array}{c}\text { Enfermedades endocrinas } \\
\text { metabólicas }\end{array}$ & Tumores \\
España & $\begin{array}{c}\text { Enfermedades del } \\
\text { aparato respiratorio }\end{array}$ & $\begin{array}{c}\text { Enfermedades del aparato } \\
\text { digestivo }\end{array}$ & $\begin{array}{c}\text { Enfermedades del } \\
\text { aparato circulatorio }\end{array}$ \\
Francia & Tumores & $\begin{array}{c}\text { Enfermedades del aparato } \\
\text { circulatorio }\end{array}$ & $\begin{array}{c}\text { Enfermedades del } \\
\text { sistema nervioso }\end{array}$ \\
Reino Unido & Trastornos & Trastornos ginecológicos & Pacientes en fase \\
& ortopédicos & terminal
\end{tabular}

pacientes (cuadro 1), siendo los tumores y las enfermedades del aparato circulatorio las dos principales causas de hospitalización en el domicilio (con excepción de Quebec, que atiende a una población muy joven) $(9,13-15)$.

En todo caso, cabe destacar que para la mayoría de los países donde se utiliza, la HD se destina principalmente a personas ancianas que necesitan cuidados paliativos y a pacientes de cáncer $\mathrm{u}$ otras enfermedades degenerativas terminales $(12,16-18)$. Los diagnósticos tratados más frecuentemente en casos de HD comprenden tumores, complicaciones posquirúrgicas, enfermedad pulmonar obstructiva crónica y sida.

En general, los países siguen el modelo francés, que impulsa paralelamente las dos modalidades conocidas de servicios de salud en el hogar: la HD y la atención primaria a domicilio (APD) (9). A propósito, la HD aparece como una modalidad asistencial distinta de la APD en los siguientes países: Canadá, España, Francia, Reino Unido y Suecia. En cambio, en Alemania y Estados Unidos, la noción de atención domiciliaria integra a la vez los dos conceptos de APD y HD (2).

Hay que destacar que a estos conceptos diferentes entre los países que optaron por introducir la HD se añade un gran vacío jurídico, ya que tan solo Alemania, Estados Unidos y Francia disponen de una definición y una reglamentación sobre la hospitalización a domicilio (9).

En España la creación de los servicios de HD fue motivada por la saturación de los hospitales y por el intento de racionalizar el gasto sanitario. Se caracterizó desde el inicio por un crecimiento anárquico, alimentado por proyectos individuales, sin un plan maestro que permitiera unificar conceptos y establecer criterios por lo menos entre las diversas comunidades autónomas (11-13).

La transferencia de competencias en materia de sanidad a las comunidades autónomas españolas contribuyó a este desarrollo desigual de la HD en todo el país. Por ejemplo, se observa una gran evolución de esta modalidad en el País Vasco y en la Comunidad Valenciana, donde además se han creado marcos regulatorios exclusivos para la HD (11, 13). En 1992, el Servicio Vasco de Salud-Osakidetsa hizo público su programa "Alternativas a la hospitalización", dando lugar a la creación y al fortalecimiento de los servicios de hospitalización a domicilio. En 1995, la Consellería de Sanidad y Consumo del Gobierno Valenciano dio a conocer una orden (DOGV n ${ }^{\circ}$ 2527) dirigida a la creación y definición de las unidades de HD en los hospitales pertenecientes al Servicio Valenciano de Salud (11). En el resto de las comuni- 
dades autónomas de España no hay normativas expresas para la HD.

A título de ilustración, en la Comunidad Valenciana, la Consellería de Sanidad y Consumo comenzó la implantación de unidades de HD en 1990 (en hospitales con características más o menos comunes en cuanto a su ámbito urbano, sobreocupación, alta carga asistencial y servicios de referencia de otros hospitales) $(19,20)$, ubicándolas en el marco hospitalario y con la misión de servir de puente entre el hospital y la atención primaria (21). Se trata, por lo tanto, de un programa que depende orgánica y funcionalmente de la estructura de asistencia especializada, teniendo como referencia el hospital de área ${ }^{9}$ (de acuerdo con el decreto 122/ 88) $(20,21)$ y articulándose como una más de las modalidades asistenciales dentro del sistema de salud $(22,23)$.

\section{Características principales de la hospitalización domiciliaria actual}

En la actualidad las modificaciones de los hábitos sanitarios, sumadas a los intensos cambios demográficos y sociosanitarios (como el incremento de la esperanza de vida de la población y el consecuente y progresivo crecimiento de la población anciana), obligan a buscar nuevas formas de gestión y propuestas de reformas estructurales que faciliten el desarrollo de medidas y reorientaciones en el sistema sanitario $(19,22)$.

Como consecuencia, en todo el mundo se han ideado y promovido numerosas opciones institucionales, ambulatorias y domiciliarias de atención de la salud. La HD, una de ellas, está adquiriendo cada vez más importancia en las distintas organizaciones sanitarias y sociales, así como en diferentes sectores de la sociedad (pacientes y familiares, administración sanitaria, profesionales y servicios sanitarios).

Dada esta realidad, la mayor parte de los estudios sobre el tema prevén

\footnotetext{
9 Área de salud es cada una de las unidades administrativas en las que está dividida la asistencia sanitaria de la Comunidad Valenciana.
}

un aumento de los servicios de atención domiciliaria y señalan que los sectores sanitario, social, político y económico sitúan la HD como una alternativa asistencial muy halagadora, viable y prometedora $(6,24-26)$.

Es oportuno destacar aquí algunas características de la HD, señalando en qué difiere de otras formas de atención a domicilio. Si se revisa la literatura nacional e internacional, se observa una gran heterogeneidad de modelos de organización de atención domiciliaria que varían de acuerdo con la orientación y directrices generales de organización que tiene el sistema sanitario de cada país, asociada a la fuente de financiación y al perfil de los pacientes (o enfermedades) a que se destinan (27-30). Los servicios de atención o cuidados a domicilio básicamente se diferencian en dos grandes grupos: hospitalización domiciliaria (HD) y atención domiciliaria desarrollada por el equipo de atención primaria (APD).

Hospitalización domiciliaria. Pese a la gran variedad de definiciones encontradas en la literatura, según la mayoría de los estudios en Europa el concepto de HD que se ajusta mejor es el prevaleciente en Francia, donde se la considera " . . una alternativa asistencial del sector salud que consiste en un modelo organizativo capaz de dispensar un conjunto de atenciones y cuidados médicos y de enfermería de rango hospitalario, tanto en calidad como en cantidad, a los pacientes en su domicilio, cuando ya no precisan de la infraestructura hospitalaria pero todavía necesitan vigilancia activa y asistencia compleja" $(11,19,31)$.

Cabe mencionar, por lo tanto, el carácter transitorio que debe tener la $\mathrm{HD}$ $(11,32)$. Es decir, que la HD debe tener una duración limitada, en tanto se produce la circunstancia del alta, sea por mejoría o cura, exitus, estabilización del proceso patológico, reingreso al hospital o transferencia al servicio de atención primaria. $\mathrm{Si}$ no se observa el carácter transitorio de la HD, o si su función se interpreta inadecuadamente, se corre el riesgo de duplicar el tratamiento, ya que la vigilancia y el seguimiento crónicos en el domicilio (salvo excepciones) deben ser responsabilidad de los servicios de atención primaria $(11,28,29)$.

En ese sentido, los pacientes indicados para ingreso en las unidades de HD son aquellos que, aun cuando no necesitan toda la infraestructura hospitalaria para su tratamiento, requieren cuidados que superan en complejidad a los prestados por la atención primaria de salud. Asimismo, la HD se realiza con pacientes que requieren cuidados médicos que no justifican su ingreso a un centro hospitalario; más bien, un equipo formado por médicos, personal de enfermería y trabajadores sociales, dotados de los medios tecnológicos necesarios, acuden regularmente al domicilio del paciente para hacer el diagnóstico y proveer el tratamiento (24).

Atención primaria domiciliaria. La atención primaria domiciliaria (APD) puede dividirse en dos grandes modelos: el modelo tradicional que rige en la mayoría de los países (en gran parte dicotómico y sin continuidad entre los servicios ofrecidos por los equipos del hospital y de atención primaria) y el modelo de atención compartida (shared care o transmural care en inglés) (5, $17,27,30$ ), que permite el establecimiento de una alianza estratégica entre los equipos de los dos principales niveles sanitarios. Estos dos tipos de modelos varían según el diseño de la estrategia organizativa y de financiación de los servicios sanitarios a los que están vinculados.

El modelo tradicional está destinado a pacientes que están incorporados a algún tipo de programa o asistencia especial de salud (puede incluir elementos de promoción, protección y rehabilitación de la salud) o que, debido a su estado de aislamiento o a sus precarias condiciones de salud, requieren asistencia general (vigilancia y seguimiento crónico en el domicilio). Este modelo excluye tratamientos y cuidados complejos $(12,31,32)$.

Dentro del modelo de atención compartida, en cambio, existe un estrecho vínculo entre los profesionales de 
atención primaria y los del hospital del área de salud. En líneas generales, los equipos de atención primaria convienen previamente con los profesionales de los hospitales acerca de la continuidad y la responsabilidad del tratamiento tras el alta precoz en hospitalización convencional o HD, definiéndose el perfil adecuado de los pacientes que deben ser incluidos en el programa de APD.

En la puesta en práctica del modelo de atención compartida es esencial que tanto los equipos de atención primaria (médicos de familia, enfermeros comunitarios, fisioterapeutas, terapeutas ocupacionales y auxiliares de enfermería) como los equipos del hospital (particularmente del servicio de HD) estén bien consolidados y tengan un buen nivel de desarrollo. Esto quiere decir que los dos niveles principales (atención primaria y hospital) deben estar adecuadamente organizados, financiados e integrados para proveer ambos tipos de atención domiciliaria $(33,34)$.

Una característica interesante de este segundo modelo estriba en que la HD debe ser solicitada por el médico de familia antes de que el paciente acuda al hospital. Este es un requisito esencial para lograr una atención de calidad. En general, desde la atención primaria se valora la idoneidad de que el paciente ingrese a la HD, sin que sea necesaria la valoración por parte de un médico especialista vinculado con el hospital $(26,35)$.

Desde este punto de vista, el modo de abordar los problemas de salud, entendidos como procesos continuos e indisgregables, depende directamente del nivel de integración y de coordinación que haya entre los equipos de atención primaria y del hospital, así como del grado de comunicación que se dé entre ambas partes (36).

Un ejemplo interesante de esta segunda forma de gestionar y ofrecer programas de atención domiciliaria es el Servicio Nacional de Salud del Reino Unido (36-38). Este servicio cuenta con un programa de atención domiciliaria de calidad manejado desde la atención primaria, cuya práctica se asemeja a los programas más comúnmente gestionados por los hospitales en otros países europeos. En el Reino Unido se han creado servicios de atención domiciliaria dotados de una infraestructura y de un sistema de financiación que garantizan el seguimiento y la continuidad del tratamiento.

También resulta interesante el papel de los médicos de atención primaria organizados en el seno de las health maintenance organizations (HMO, organizaciones de servicios médicos prepagados) (39) de Estados Unidos, dado que se trata de un enfoque novedoso de la gestión sanitaria que reafirma la función importante del profesional de la atención primaria. En el marco de las HMO, a los médicos de atención primaria se les considera agentes intermediarios entre el usuario y el hospital.

Se podría argumentar que, tanto en el caso de la reforma del Servicio Nacional de Salud del Reino Unido como en el de las HMO norteamericanas, la atención primaria pasa a ocupar un espacio distinto del tradicional entre las organizaciones sanitarias. Es decir, los grupos de médicos de los servicios de atención primaria pasan a ser los responsables directos de los usuarios del sistema de salud, actuando como agentes intermediarios que encaminan, indican y compran los tratamientos y procedimientos que juzguen más adecuados para los pacientes $(34,40)$.

\section{Importancia de la hospitalización domiciliaria}

En lo que se refiere a los distintos aspectos de su funcionamiento (tales como el ámbito sanitario donde se debe desarrollar, su eficacia económica y el perfil de la población atendida), el tema de la atención domiciliaria aparece en muchos estudios como polémico y sujeto a discusiones y controversias (16, $21,22)$. No obstante, la mayor parte de los autores resaltan la necesidad de una mayor colaboración entre los servicios hospitalarios y extrahospitalarios (atención primaria, ambulatoria y domiciliaria y servicio social) para posibi- litar una asistencia continua y eficaz $(11,23,24)$. Dicho de otra forma, es imperativo adoptar una nueva filosofía asistencial que busque modernizar los sistemas sanitarios, procurando convertirlos en modelos integrados de salud basados en una responsabilidad compartida $(5,25,26,41)$.

Este argumento pone de relieve la importancia de la HD como puente entre el hospital y la atención primaria de salud, posibilitando el establecimiento de labores compartidas y permitiendo una interacción progresiva y real entre los dos principales niveles de atención (42-44).

En el campo de la organización del cuidado de la salud, la atención domiciliaria representa una posibilidad de crear un modelo consensuado y armónico de organización y financiación entre los diferentes niveles del sistema de salud, procurando lograr el establecimiento y la consolidación de servicios que respondan adecuadamente a las necesidades del usuario (45). De allí el gran interés que han despertado en los últimos años los servicios de atención domiciliaria $(9,11,46)$.

Asimismo, el rápido aumento de la implementación de unidades de HD se debe más que nada al propósito de encontrar el mejor lugar posible para el tratamiento del paciente, mejorando la calidad de su vida y utilizando más eficientemente los recursos del hospital $(12,29,47)$. En el cuadro 2 se presentan las ventajas principales que según la literatura justifican la elección de la HD.

En general las razones del interés que despierta la HD tienen sustento en dos puntos de vista. Por un lado están las consideraciones sobre efectividad. Se reconoce que el sistema de HD suele traducirse en una mejor atención, así como en mayor satisfacción y participación por parte del paciente y sus familiares durante el tratamiento. La HD también se asocia con una mejor evolución clínica en el caso de algunas enfermedades, con la reducción de ciertas complicaciones derivadas de la estancia hospitalaria prolongada o innecesaria, y con una asistencia más humana $(4,18,47,48)$. 
CUADRO 2. Ventajas de la hospitalización domiciliaria

\begin{tabular}{|c|c|c|}
\hline Pacientes y sus familiares & Hospital & Sistema de salud \\
\hline $\begin{array}{l}\text { Mejor atención, participación } \\
\text { y responsabilización }\end{array}$ & $\begin{array}{l}\text { Mayor rentabilidad de sus } \\
\text { recursos disponibles }\end{array}$ & $\begin{array}{l}\text { Incremento de la eficacia y } \\
\text { efectividad mediante una } \\
\text { asistencia más humanizada }\end{array}$ \\
\hline $\begin{array}{l}\text { Mejor calidad de vida, } \\
\text { intimidad y bienestar }\end{array}$ & $\begin{array}{l}\text { Aumento en rotación y } \\
\text { disponibilidad de camas }\end{array}$ & $\begin{array}{l}\text { Uso más eficiente de los } \\
\text { recursos sanitarios }\end{array}$ \\
\hline $\begin{array}{l}\text { Asistencia personalizada } \\
\text { y más humanizada }\end{array}$ & $\begin{array}{l}\text { Reducción de estancias } \\
\text { innecesarias }\end{array}$ & $\begin{array}{l}\text { Posibilidad de integrar y mejorar } \\
\text { las relaciones entre los } \\
\text { diversos niveles asistenciales }\end{array}$ \\
\hline Educación para la salud & $\begin{array}{l}\text { Acortamiento de los períodos } \\
\text { de internamientos }\end{array}$ & $\begin{array}{l}\text { Posibilidad de atención } \\
\text { continuada, integral y } \\
\text { multidisciplinaria entre los } \\
\text { profesionales de atención } \\
\text { primaria, hospitalaria y social }\end{array}$ \\
\hline $\begin{array}{l}\text { Prevención de desinserción } \\
\text { social y hospitalismo psíquico }\end{array}$ & Reducción presupuestaria & \\
\hline Menor riesgo de yatrogenia & $\begin{array}{l}\text { Posibilidad de mejorar los } \\
\text { servicios }\end{array}$ & \\
\hline
\end{tabular}

Por otro lado, diferentes autores han argumentado que la HD, al permitir un uso más adecuado y racional de los recursos, tiende a disminuir el gasto en salud $(9,22,39,49)$. La práctica de la atención en el domicilio aumenta la disponibilidad de camas y acorta los períodos de internamiento en los hospitales. Adicionalmente, los costos de agregar camas en la HD es menor que las inversiones necesarias para crear camas hospitalarias o para la ampliación o construcción de hospitales, aparte de que el costo por estancia es menor en la HD que en la hospitalización tradicional $(4,40,50,51)$.

Como regla general, la decisión de ingresar un paciente en el programa de HD, además de basarse en el cumplimiento de algunos requisitos, se adopta en consonancia con dos grandes modelos (cuadro 3) $(26,34,35)$. Un primer modelo está determinado por la procedencia de los pacientes, donde se incluyen aquellos grupos de pacientes que tienen la posibilidad de ser dados de alta tras un período de hospitalización convencional de corta duración, seguido por una HD y posteriormente un alta definitiva. Dentro de este grupo figuran, por ejemplo, los pacientes de intervenciones quirúrgicas traumatológicas o digestivas. Un segundo modelo agrupa a los pacientes que provienen de la comunidad y que, si no existiese el servicio de HD, serían ingresados en el hospital tradicional. Incluye, por ejemplo, pacientes afectados por neumonías o accidentes cerebrovasculares. El procedimiento más adecuado para que se ingrese el paciente en HD según este modelo debería seguir el siguiente orden: domicilio $\rightarrow$ médico de familia y/o servicio de urgencia $\rightarrow$ admisión en HD $\rightarrow$ alta definitiva y/o referencia al médico de familia (atención primaria).

Sin embargo, si bien en principio no debería haber una lista previa de perfiles y diagnósticos de pacientes candidatos a ser ingresados en una unidad de HD (lo más adecuado sería aplicar un criterio basado en las características epidemiológicas y sociodemográficas de la población de referencia y del hospital o centro de salud del área sanitaria), algunos estudios clasifican a los servicios de HD según los trastornos más susceptibles de ser incluidos en estos tipos de programas. Según la literatura, los pacientes más frecuentes en $\mathrm{HD}$ son $(12,26,43)$ :
- pacientes con situaciones agudas: enfermedades infecciosas, neumonías, pielonefritis, aplasias tras la quimioterapia y antibioticoterapia domiciliaria por vía intravenosa, enfermedades vasculares, enfermedades neurológicas, pacientes con nutrición parenteral y ventilación mecánica a domicilio;

- pacientes con procesos crónicos reagudizados: EPOC (enfermedad pulmonar obstructiva crónica), cardiopatías, hepatopatías crónicas, sida (síndrome de inmunodeficiencia adquirida), cirrosis hepática, neoplasias y enfermedades terminales;

- pacientes con procesos quirúrgicos: postoperatorio inmediato de cirugía menor y media, postoperatorio tardío y complicaciones de cirugía mayor, postoperatorio de cirugía traumatológica y ortopédica, y el tratamiento de grandes escaras y úlceras cutáneas.

\section{Problemas operacionales y gerenciales encontrados en la HD}

Pese a que desde hace algunos años vienen confluyendo una serie de factores que hacen que las diferentes organizaciones sanitarias y sociales consideren la HD como una alternativa asistencial interesante, el consenso se pierde a la hora de diseñar estrategias organizativas y de financiación de estos servicios. La idea es superar las deficiencias y alcanzar los retos relacionados con los problemas de comunicación, integración y coordinación a que se hayan sometidos los servicios de atención primaria y hospitalaria (50-52).

Diversos estudios $(14,53,54)$ señalan que el modelo organizativo vigente en la mayoría de los países, centrado en las direcciones y gestionado mediante programas desintegrados y organizados mucho más en función de las características estructurales de los servicios y las instituciones que de las necesidades reales de la población de referencia, es la causa principal de las deficiencias y dificultades de la asistencia sanitaria en alcanzar una cobertura satisfactoria. En ese sentido, uno de los mayores retos que se plantean en la actualidad consiste en transfor- 
CUADRO 3. Requisitos para la inclusión de pacientes en programas de atención domiciliaria

Aceptación voluntaria del paciente y la familia tras recibir la información adecuada sobre el programa y el tipo de atención en el domicilio.

Existencia de una estructura familiar que pueda asumir la responsabilidad del cuidado activo del paciente en el domicilio -o un profesional especializado contratado para tal fin.

Las enfermedades susceptibles de atención domiciliaria (excepto pacientes con enfermedades crónicas, en fase avanzada y terminales) tienen que ser transitorias y limitadas en el tiempo. Deben existir momentos de ingreso y de alta.

La vivienda debe poseer ciertos requisitos mínimos en su estructura física, de modo que no pueda actuar como factor de riesgo de complicaciones.

Se deben proporcionar los recursos técnicos y de otro tipo necesarios para que los pacientes reciban cuidados de calidad.

El servicio de atención domiciliaria debe estar bien coordinado e integrado con los otros servicios sanitarios (hospital, servicios de urgencias, servicios sociales y atención primaria).

mar la tradicional y rígida jerarquización de los servicios de salud por niveles asistenciales, en un nuevo modelo que elimine las barreras físicas de las organizaciones sanitarias, impulsando a la adopción de una nueva filosofía de asistencia integrada y compartida entre la atención prestada por el centro de salud y el hospital $(14,55,56)$. Es justo ahí donde la atención domiciliaria encuentra todo su significado y puede demostrar que constituye una herramienta eficiente.

No obstante, en los campos operacional y gerencial de los servicios de HD la evidencia empírica señala que hay numerosas circunstancias que han contribuido a acrecentar las dificultades para implementar medidas que proporcionen una mayor comunicación e integración entre los dos niveles sanitarios. Entre ellas se destaca el déficit de experiencia en la gestión de organizaciones en red y direcciones de proyectos comunes por parte de los gerentes de las diferentes organizacio- nes sanitarias, la necesidad de dedicar gran cantidad de tiempo - no siempre disponible para los profesionales y gerentes- y la fragmentación y superposición de servicios. Todas estas circunstancias están además asociadas a la falta de una coordinación adecuada y un nivel superior de autoridad que se encargue de brindar incentivos para que tanto la atención especializada como la atención primaria (y el servicio social) converjan hacia un modelo de atención compartida $(6,27,28,57)$.

Desde esa perspectiva, existe coincidencia en el diagnóstico de las deficiencias en los resultados de los servicios ofrecidos tanto por la atención primaria como por el hospital. Por un lado, la atención primaria es criticada por su incapacidad para reducir las urgencias hospitalarias, por la variabilidad en la oferta asistencial entre los distintos centros, por las derivaciones innecesarias al ámbito hospitalario y por su limitada capacidad para contener el acceso a la atención hospitalaria ya que se encuentra sobresaturada por su propia demanda $(32,33,57)$. Por otro, la atención hospitalaria es criticada por presentar un modelo transversal, vertical y centrado en la enfermedad, careciendo de una debida y necesaria integración sociosanitaria del paciente, y de un abordaje más humanizado y personal de la asistencia $(33,58,59)$.

En consecuencia, el sistema de prestación de servicios domiciliarios carece de un enfoque integral y comunitario de los problemas de salud, más allá de la suma de los problemas individuales y de la atención prestada por cada nivel sanitario $(4,14,58,60)$. De ahí que los diferentes colectivos coincidan en que el funcionamiento del conjunto del sistema sanitario prescinde del establecimiento de una interrelación armónica entre los dos ámbitos esenciales del sistema (atención primaria y hospital), basada en relaciones de igualdad y en la delimitación clara de sus funciones. Es necesario buscar puntos de colaboración y coordinación entre los dos niveles sanitarios, que sean a la vez valorados positivamente tanto por la atención primaria como por el hospital, sin ser percibidos como imposiciones o invasiones técnicas de poder de uno sobre el otro $(61,62,63)$.

De hecho, ante todas las dificultades identificadas, la atención domiciliaria plantea ciertos problemas de competencia $(16,22,24)$ frente a las diferentes organizaciones que ofrecen servicio (ocasionando no raras veces la duplicación de servicios en la comunidad o la creación de una red asistencial paralela). Esta situación crea cierta tensión entre profesionales y niveles asistenciales, llevando a círculos viciosos difíciles de controlar desde la forma como se estructura la organización sanitaria actual $(9,25,28,64)$. Cabe mencionar que tanto la atención primaria como la atención hospitalaria tienen su espacio en el ámbito domiciliario. Se comprende que cada nivel tiene sus propias limitaciones, pero cuando ambos pueden desarrollar su capacidad potencial se forma un conjunto altamente efectivo (46).

En este contexto, se debe destacar la urgente necesidad de implementar medidas prácticas y estratégicas de colaboración y asistencia compartidas entre hospital y atención primaria, que incidan directamente en los puntos críticos del funcionamiento de los programas de atención domiciliaria. De este modo se plantea el desafío de innovar esquemas que permitan sumar, incluir e integrar (y no lo contrario, como suele ocurrir). Es perentorio profundizar en el diseño de un modelo consensuado de organización y de financiación (en un proceso de atención continuado, integral y multidisciplinario), desarrollando así una política decidida que permita el establecimiento de una alianza estratégica (elemento prioritario y esencial para la mejoría de los procesos asistenciales) entre ambos servicios $(6,24,32,33)$.

\section{Propuestas para una gestión innovadora y exitosa en HD}

En la práctica los sistemas de salud modernos, inspirados, por ejemplo, en los modelos británico y holandés (32, $33,59)$, se orientan hacia un nuevo escenario donde la integración de todas las funciones sanitarias y la coordina- 
ción entre los distintos niveles asistenciales deben generar un sistema de información de flujo bidireccional (63-65). Este es un requisito para el modo en que se hace frente a todos y cada uno de los problemas de salud, que pasan a ser entendidos como procesos continuos e indisgregables (52, 60, 66).

Con esta idea, a continuación se presentan algunas propuestas concretas de colaboración entre el hospital y la atención primaria en el ámbito asistencial. Tales propuestas pueden estar referidas directamente a la HD o a servicios que indirectamente contribuyan al éxito de esta modalidad asistencial. Hay que destacar la importancia concedida al papel que puede ocupar la atención domiciliaria en la reorientación y reforma de los sistemas y servicios de salud hacia una sanidad moderna, efectiva y de calidad a toda la población.

\section{Propuestas intrínsecas a la HD}

- Cambiar las estrategias tradicionales de prestación de servicios que caracterizan a los hospitales $(58,62,68)$ (instituciones cerradas, con una prestación de servicios vertical y unidireccional), mediante el replanteamiento de objetivos, organización, actividades y servicios médicos-asistenciales ofrecidos, en función de las nuevas estrategias sanitarias y de las actuales demandas sociales, demográficas y de gestión (67-69).

- Fortalecer o crear canales y mecanismos de comunicación interinstitucional entre los niveles sanitarios (y también entre los servicios sociales de la comunidad) $(59,62,70)$, a modo de garantizar el intercambio de información, la integración de las actividades y la continuidad del proceso asistencial. Se debe incrementar la colaboración entre hospital y atención primaria, reduciendo brechas de comunicación y facilitando la transmisión de datos referidos a los procesos asistenciales y de los pacientes. Este proceso implica dotar a las instituciones de salud de equipos y sistemas de información modernos y confiables, que permitan tomar decisiones clínicas con la mayor certidumbre posible y continuar la atención de los pacientes en cualquiera de los puntos y niveles del sistema sanitario $(30,34,58)$.

- Diseñar (en conjunto) protocolos o guías de actuación (preventiva, diagnóstica, terapéutica, de transmisión de datos clínicos, de seguimiento y control) (71-73) entre los dos niveles de un área de salud. Tales medidas deben ir acompañadas de la designación de los profesionales (en el hospital y en la atención primaria) que serán responsables en este sentido $(20,62)$, generando así una cultura de corresponsabilización en las decisiones sobre el tratamiento del paciente. Estas medidas pueden contribuir decisivamente a mejorar la calidad y la efectividad de los procesos asistenciales al implementar los enfoques integrales del paciente ingresado, disminuir las posibilidades de errores por cambios inadecuados de tratamientos y evitar conflictos interprofesionales o daños por repeticiones innecesarias de pruebas y exploraciones $(26,30)$. Hay procesos donde el delineamiento de protocolos y programas de actuación conjunta son especialmente indicados, entre ellos la atención a los pacientes dados de alta "precozmente" con la consecutiva planificación del alta hospitalaria, y el cuidado de pacientes con enfermedades terminales o cuyas afecciones son mejor tratadas mediante la continuación del tratamiento en el domicilio $(73,74)$.

\section{Propuestas extrínsecas a la HD}

- Establecer o fortalecer el área de salud como eje fundamental del sistema de atención $(24,25)$, teniendo en el centro de salud el punto de referencia para cualquier problema sanitario individual o de la comunidad, donde concretamente se pongan en práctica los principios de integración y coordinación de los recursos, servicios y actividades. Hay que avanzar siempre hacia la municipalización del sistema de salud $(49,59)$.

- Fortalecer la atención primaria $(58,62$, 68), buscando recobrar y consolidar el prestigio frente a la población y la comunidad sanitaria mediante modificaciones de la estructura y la lógica de prestación de los servicios. Tales cambios permitirían, por ejemplo, ofrecer consultas no masificadas, aumentar progresivamente las consultas programadas, brindar atención continuada, adquirir mayor permeabilidad a las nuevas orientaciones de salud y revitalizar la labor clínica $(58,59)$.

- Invertir en una buena formación de recursos humanos, a fin de lograr una real implicación de los profesionales sanitarios en la prestación de servicios y la profesionalización de los gerentes y planificadores sanitarios. La formación de recursos humanos debe ser amplia y abarcar desde la formación de pregrado - donde los estudiantes de medicina y de enfermería deban rotar por centros de salud y hospitales en actividades prácticas y curriculares- $(62,70)$ hasta los programas de educación médica de postgrado $(32,50)$. En este último caso habría que implementar la especialidad de "medicina de familia y comunitaria" (por tratarse del profesional aglutinador, con la visión global del paciente y de su tratamiento), la cual debería contener períodos de aprendizaje en los niveles de atención primaria y hospitalario. Adicionalmente, la educación sanitaria debe ofrecer programas de formación continuada de calidad $(38,71)$, los cuales constituyen instrumentos útiles, tanto para potenciar la coordinación e integración entre los dos niveles como para favorecer el establecimiento de vínculos entre profesionales del hospital y la atención primaria, facilitando de esta manera la realización de interconsultas y la programación conjunta de otras actividades docentes y de investigación.

- Impulsar las funciones docente e investigadora (además de la función asistencial), las cuales deben coexistir en los 
centros de salud y hospitales $(56,62$, 75). Un componente clave en la formación de recursos humanos de calidad y en el establecimiento de programas de pregrado y posgrado es la participación de las universidades. Es preciso estimular tal participación, creando polos de capacitación, formación y educación continuada y colaborando en la formulación y aplicación de protocolos de actuación y derivación comunes entre la atención primaria y especializada.

- Institucionalizar la figura del médico consultor especialista, como "puente" entre el hospital y la atención primaria. Este profesional puede ayudar al mejor entendimiento entre ambos niveles, en la línea de la integración bidireccional y actuando como agente facilitador en el ámbito de la coordinación del sistema $(27,30,62)$.
- Construir alianzas estratégicas entre las partes del sistema sanitario y los servicios sociales, que proporcionen una mayor convergencia de las políticas sanitarias y las sociales en beneficio del paciente, superando la siempre presente provisión dicotómica entre los diferentes servicios y profesionales que participan en el tratamiento. En este contexto se inscribe la atención domiciliaria, y en especial la hospitalización a domicilio, donde la calidad de la atención ofrecida está estrechamente ligada a la existencia de una alianza estratégica entre los equipos sanitarios (de atención primaria y de atención hospitalaria) y sociales $(55,63,68,75)$.

Finalmente se puede concluir que la HD, en cuanto alternativa a la hospitalización tradicional, conlleva el benefi- cio adicional de hacer posible y tornar viable el establecimiento de una innovadora y necesaria coalición y alianza estratégica en el campo de la salud pública y la administración sanitaria. De hecho, exige un mayor compromiso y más responsabilidad por parte de todos los actores involucrados en el proceso, incluidos los gerentes de centros de salud, los médicos y otros profesionales sanitarios, los pacientes y sus familiares, y la comunidad local.

Agradecimiento. El contenido de este artículo se basa en investigaciones que fueron financiadas por el Conselho de Desenvolvimento Científico e Tecnológico (CNPq)/Brasil, y forma parte de un esfuerzo conjunto entre Brasil y España dirigido a explorar gestiones innovadoras en los sistemas sanitarios.

\section{REFERENCIAS}

1. Saltman RB, Figueras J. European Health Care Reform. Analysis of current strategies. Copenhagen: WHO, Regional Office for Europe; 1997.

2. Brodsky J, Habib J. New developments and issues in home care policies. Disabil Rehabil 1997;19(4):150-154.

3. Neuhauser D. Home care, medical care and the new competitive environment. Disabil Rehabil 1997;19(4):155-157.

4. Fuente DOF, Ridao M, Peiró S, Marchan C. Hospitalización a domicilio y hospitalización convencional: una evaluación económica. Med Clin (Barc) 1997;109:207-211.

5. Pritchard P, Huges J. Shared care. The future imperative? London: Royal Society of Medicine Press; 1995.

6. Contel SJC. La atención a domicilio como modelo de atención compartida (editorial). Atención primaria 2000;25(8):22-28.

7. Anand JK, Miles JW. Hospital at home which way will it go? JR Soc Med 1997;90(7):414-5.

8. Stessman J, Hammerman-Rozenberg R, Cohen A. Home hospitalization in the spectrum of comunity geriatric care. Disabil Rehabil 1997; 19(4):134-141.

9. Raffy-Pian N. La hospitalización a domicilio: un lugar marginal dentro de los sistemas sanitarios. Rev Gerontol 1997;7:5-9.

10. Castillo JR. Análisis del origen, situación y perspectivas de futuro del proceso de descentralización sanitario español. Rev Española Salud Publica 1998;72(1):1-15.
11. Glez MDD (coord). Hospitalización a domicilio. España: Hoechst Marion Roussel; 1998.

12. González GG, Cueto-Felgueroso J, García CA, Menéndez AG. La hospitalización a domicilio: una alternativa a la hospitalización convencional. Medicina Integral 1995;25(4): 151-155.

13. Colomer J, González MJI, González RVJ. Alternativas a la hospitalización: una respuesta lógica al aumento de la demanda. En: Del Llano J, Ortún V, Millán J, Gené J, eds. Gestión sanitaria: innovaciones y desafíos. Barcelona: Masson 1998;359-401.

14. Stewart S, Marley JE, Horowitz JD. Effects of a multidisciplinary, home-based intervention on unplanned readmissions and survival among patients with chronic congestive heart failure: a randomised controlled study. Lancet 1999;354(25):1077-1083.

15. Cline $C$, Israelson $B$, Willenheimer $R$, et al. A cost effective management programme for heart failure reduces hospitalisation. Heart 1998;80:442-446.

16. Pardo C MR, et al. Papel de los equipos de atención primaria en la hospitalización infantil de los niños menores de 2 años. Atención Primaria [serial online] 2001 Ene [cited 2000 oct 31];26(7) [7 screens]. www.db.doyma.es/.

17. Shepped S, Ilife S. Effectiveness of hospital at home compared to in-patient hospital care. En: Bero L, Grilli R, Grimshaw J, Osman A, eds. Collaboration on effective professional practice module of the Cochrane Database of
Systematic Reviews. The Cochrene Collaboration Issue 1. Oxford: Updated Software; 1998.

18. Coleman B. European models of long-term care in the home and community. Int J Health Serv 1995;25:455-474.

19. Martín AP, García FJC, Correa ME, et al. Factores de riesgo de ingreso hospitalario en mayores de 64 años. Gaceta Sanitaria 2000;14(5): 363-370.

20. Contel SJC. La hospitalización a domicilio no es una alternativa plenamente razonable. Atención Primaria 1999;24(3):162-168.

21. Mediano C, Guillén M, Aranda E, Pérez F, Peiró S. Como en casa en ningún sitio. Satisfacción de los pacientes hospitalizados a domicilio. Revista Calidad Asistencial 1995;1: 13-18.

22. López FAA, coord. La informatización de atención primaria (yII). Atención Primaria [serial online] 2001 Ene [cited 2000 nov 15]; 26(8) [35 screens]. www.db2.doyma.es/

23. Cowen ME, Dusseau DJ, Toth BG, Guisinger C, Zodet MW, Shyr Y. Casemix adjustment of managed care claim data, using the clinical classification for health policy research method. Med Care 1998;36:1108-1113.

24. LoFaso V. The doctor-patient relationship in the home. Clin Geriatr Med 2000;16(1): 83-94.

25. Kellogg FR, Brickner PW. Long-term home health care for the impoverished frail homebound aged: a twenty-seven-year experience. J Am Geriatr Soc 2000;48(8):1002-1011. 
26. Raffy-Pihan, N. "L'hospitalitasion à domicile. Un tour d'horizon en Reurope, aux Etats-Unis et au Canada". CREDES; 1994.

27. Orton V. Shared care. Lancet 1994;1.413-1.414.

28. Segura Noguera JM, et al. Enfermos crónicos domiciliarios y consumo de psicofármacos. Atención Primaria [serial online] 2001 Ene [cited 2000 nov 30];26(9) [6 screens]. www.db. doyma.es/

29. Van der linder BA. Transmural care: facts and future: En: Schrijvers AJP, ed. Health and health care in the Netherlands. Utrecht: De Tijdstroom; 1997.

30. Hardy B, Mur-Veemanu I, Steenberger M, Wistow G. Inter-agency services in England and the Netherlands: a comparative study of integrated care development and delivery. Health Policy 1999;48:87-105.

31. Noguera JMS, Montaña MCB, Bastú NB, Gastalver DC. Enfermos crónicos domiciliarios: valoración integral físico-cognitiva y caídas durante 3 años de seguimiento. Atención Primaria 2000;25(5):35-38.

32. Badia JG. ¿El hospital, debe competir, dominar o compartir con la atención primaria? Atención Primaria 1998;21(4):16-17.

33. Ortúm V. La articulación entre niveles asistenciales. En: Del Llano J, Ortúm V, Martín JM, Milán J, Gené J, eds. Gestión sanitaria: innovaciones y desafíos. Barcelona: Masson; 1998.

34. Davies TM. Future challenges for the general practitioner: hospital at home. En: The Royal College of General Practitioners Member's Reference Book. London: Campden Pub.; 1997.

35. North West Anglia Healthcare Trust. Hospital at home: a new way of caring. Peterborough: North West Angia Healthcare Trust; 1995.

36. Lefebvre A. L'hospitalisation à domicilie. Soins 1996;609:6-9.

37. Ham C. Replacing the NHS market. The white paper should focus on incentives as well as directives. Br Med J 1997;315:1.175-1.176.

38. Riera JMC, Oliver JG, Ballart, JDF, et al. Opinión de los médicos de atención primaria acerca de su hospital de referencia. Todo Hospital 1997;140:7-12.

39. Fuente DO, Moreno SP, Rodríguez CM, López MR. Cuidados de enfermería en hospitalización a domicilio y hospitalización convencional. Rev Española Salud Publica [serial online] 2001 [cited 1998];72(6)[13 screens]. www.scielosp.org/

40. Moreno JM, Gomis P, Valero MA, León-Sanz M. Nutrición parenteral domiciliaria: experiencia de un programa combinado (adultosniños) a lo largo de 6 años. Med Clin Barc 2000;114(16):617-618.

41. Jones J, Wilson A, Parker H, Wynn A, Jagger C, Spiers N, Parker G. Economic evaluation of hospital at home versus hospital care: cost minimisation analysis of data from randomised controlled trial. BMJ 1999;319:1547-1550.

42. Conferencia Internacional sobre Atención Primaria de Salud. Alma-Ata, URSS. Septiembre de 1978. Ginebra: OMS; 1978.

43. Lippeveld T, Sauerborn R, Sapiri S. Por unos sistemas de información sanitaria eficaces.
Foro Mundial de la Salud. Ginebra: OMS; 1997;18:196-205.

44. Bermejo GB, Angulo KL, Agorria AA, Barruetabeña MS, Arrate AO. Características socioeconómicas, problemas y necesidades de salud de los pacientes crónicos domiciliarios. Atención Primaria 1997;20(5):230-236.

45. Matezans R. Integración entre niveles asistenciales. Jano 1999; LVI (1285):12.

46. Alessi CA, Stuck AE, Aronow HU, Yuhas KE, Bula CJ. The process of care in preventive inhome comprehensive geriatric assessment. Jam Geriatr Soc 1997:45:1044-1050.

47. Generalitat Valenciana (Conselleria de Sanitat i Consum). Hospitalización a domicilio; 1996 (fotocopia).

48. Cuerda MDC, Castelló FB, Giménez MLB. Coordinación en el sistema sanitario: algunos ejemplos. Gestión Hospitalaria 1991;78:27-32.

49. Hospitalización a domicilio y enfermería: datos y reflexiones tras las primeras experiencias en España. Labor Hospitalaria 213.

50. Estéves PMH, Santos GAR, Brito EN, Rosario MAB, Freire GG. Propuesta de un modelo de organización de atención domiciliaria basada en atención primaria. Cuadernos de gestión 1999;5(1):31-38.

51. Newbury J, Marley J. Preventive home visits to elderly people in the community. Visits are most useful for people aged $>/=75$. British Med J 2000;321:19-26.

52. Crome P, Malham A, Baker D, Smith AE, Bloor R. Domiciliary visits to the old and the mentally ill: how valuable? J R Soc Med 2000; 93(4):187-190.

53. Llife S. Hospital at home: from red to amber? Br Med J 1998;316:1761-1762.

54. Coast J, Richards SR, Peters T, Gunnell D, Darlow MA, Pounsford J. Hospital at home or acute hospital care? A cost minimisation analysis. Br Med J 1988;316:1802-1806.

55. Fuente DO, Moreno SP, Rodríguez CM López, MR. Cuidados de enfermería en hospitalización a domicilio y hospitalización convencional. Rev Española Salud Publica [serial online] 2001 [cited 1998];72(6)[13 screens] www.scielosp.org/

56. Hadley J, Rabin D, Epstein A, Stein S, Rimes C. Posthospitalization home health care use and changes in functional status in a Medicare population. Med Care 2000 May; 38(5): 494-507.

57. Generalitat Valenciana. Salud y sistema sanitario (territorio, formación, inmigración, sistema de información ARGOS. Revista Valenciane D'Estudios Autonomics 1999;28:3-51.

58. Segura JCC. La atención a domicilio como modelo de atención compartida (editorial) Atención Primaria 2000;25(8):1-5.

59. OPAS/OMS- Escritório de Representação no Brasil. A saúde no Brasil [monograph online]; 1998.

60. Kaster W, Streit ME. Institutional economic: social order and public policy. Cheltenham: Edward Elgar; 1999.

61. Zurro AM. Compartir y coordinar la atención de los pacientes entre el hospital y la atención primaria: algunas propuestas prácticas (Editorial). Atención Primaria 2000;26(2):1-4.

62. Gregandi MS, Hanson LC. Comprehensive geriatric assessment: Where do we go from here? Arch Med 1996;156:15-17.

63. Bernabei R, Landi F, Gambassi G, Sgaradi A Zuccala G, Mor V, et al. Randomized trial of impact of model of integrated management for older people living in the community. $\mathrm{Br}$ Med J 1998;316:1348-1351.

64. Batztán JJ, Gil L, Andrés E. Actividad comunitaria de un servicio de geriatría hospitalario: un ejemplo práctico de coordinación entre atención primaria y especializada. Atención Primaria 2000;26(06):347-382.

65. Hutten JBF, Kerkstra A. Home care in Europe. Vertmont: Ashgate Pub Co.; 1996.

66. Kellogg FR, Brickner PW. Long-term home health care for the impoverished frail homebound aged: a twenty-seven-year experience. J Am Geriatr Soc 2000;48(8):1002-1011.

67. Segura CJC. Hacia un modelo de atención a domicilio. Cuadernos de Gestión 1999;5(1): $1-4$.

68. Hernández J Martínez. Evolución en las relaciones entre la asistencia primaria y la asistencia especializada. Todo Hospital 1999;65: $17-23$.

69. Maya, MC, et al. El conocimiento de la actividad asistencial: ¿otro factor profesional relacionado con la utilización? Atención Primaria 2000;25(7):1-2

70. Schraiber LB, et al. Planejamento, gestão e avaliação em saúde: identificando problemas. Ciencia \& Saúde Coletiva 1999;4(2):221-242.

71. Llordachs F, Kase K, Sullà E. Reflexions sobre les aliances estratègiques en el sistema sanitari. Full Econòmics 1998;32:6-12.

72. Boling PA. The value of targeted case management during transition care. JAMA 1999; 281:656-657.

73. Gravil JH, Al-Rawas OA, Flanigan V, et al. Home treatment of exacerbations of chronic obstructive pulmonary diasease by an acute respiratory assessment service. Lancet 1998; 1853-1855.

74. Naylor MD, Brooten D, Campbell R, Jacobsen BS, Mezey MD, Pauly MV, et al. Comprehensive discharge planning and home follow-up of hospitalized elders. A randomized clinical trial. JAMA 1999;281:613-620.

75. Steiner A, Walsh B, Pickering RM, et al. Therapeutic nursing or unblocking beds? A randomised controlled trial of a post-acute intermediate care unit. Commentary: Problems with randomised consent. Authors' reply. British Medical Journal 2001;322:7284

Manuscrito recibido el 13 de marzo de 2001. Aceptado para publicación, tras revisión, el 11 de junio de 2001. 
ABSTRACT Objective. To establish the different forms that the concept of home care has taken over time as a modality for providing health care, so as to contribute to the debate on the circumstances affecting health care management in the face of future challenges.

Home hospitalization: Methods. The literature is reviewed on home care as a method for delivering health care, by researching publications indexed during the period of 1995-2000 in three medical bibliographical databases: MEDLINE, LILACS (Latin American and Caribbean Health Sciences) and the Índice Médico Español (Spanish Medical Index).

Results. Home hospitalization has developed differently over time in various countries, with an assortment of models for this type of care, each with its respective advantages and disadvantages.

Conclusions. There are some criteria and proposals that could provide a framework for home care that is innovative, effective, and of high quality. Home care could help in the design and creation of a consensus on an organizational and financing model that fits in harmoniously between primary care and care in a hospital.

\title{
Convención Internacional "Salud Pública 2002"
}

Fechas: 1 a 5 de mayo de 2002

Lugar: La Habana, Ouba

日 Comité Organizador de la Convención Internacional "Salud Pública 2002" se complace en anunciar las actividades que se realizarán en el marco de la Convención, que será celebrada en el Palacio de las Convenciones de La Habana. 日 principal propósito de este importante encuentro internacional es fortalecer la colaboración y el intercambio científico entre los especialistas de distintos países dedicados a las diferentes ramas de la salud pública, a fin de analizar de manera integral las crisis de la reforma de los sistemas de salud y examinar los nuevos retos de la salud pública en el siglo XXI. Se abordarán temas muy heterogéneos; recursos humanos, nutrición, salud escolar, inmunización, epidemiología clínica y genética, educación pública, economía de la salud, informática, mortalidad infantil, atención primaria, "globalización" económica, promoción de la salud y muchos más.

日 evento tiene el apoyo del Ministerio de Salud Pública de la República de Cuba, así como de la OPS, la OMS y muchas otras entidades de salud pública cubanas e internacionales. Habrá conferencias, mesas redondas y paneles de discusión, además de presentaciones de pósteres o carteles sobre temas libres. $日$ programa comprende cursos y visitas a instituciones científicas en Giudad de La Habana.

\author{
Información: \\ Dr. Pedro Más Bermejo \\ Presidente, Comité Organizador \\ Tel.: (537) 786755 \\ Fax: (537) 662404 \\ Correo electrónico: director@inhem.sld.cu \\ Dra. Miriam Martínez Valladares \\ Vicepresidente, Comité Organizador \\ Tels.: (537) 333687 y 553324 \\ Fax.: (537) 662312 \\ Correo electrónico: miriam@hesp.sld.cu \\ Lic. Alicia García González \\ Organizadora Profesional \\ Palacio de las Convenciones de La Habana \\ Tels.: (537) 285199 y 226011 al 19 ext. 1514 \\ Fax.: (537) 228382/287996/283479 \\ Correo electrónivco: aliciag@palco.get.cma.net
}

